\title{
Pressure Effects in the Iron Chalcogenides
}

\author{
M. Bendele $\cdot$ E. Pomjakushina $\cdot$ K. Conder . \\ R. Khasanov $\cdot$ H. Keller
}

Received: 19 September 2013 / Accepted: 28 September 2013 / Published online: 14 November 2013

(C) Springer Science+Business Media New York 2013

\begin{abstract}
In $\mathrm{FeSe}_{1-x} \mathrm{Te}_{x}$, the superconducting and magnetic properties can be tuned not only by the Se-Te ratio $x$, but also by application of hydrostatic pressure. It was seen that both parent compounds FeSe and FeTe are extremely sensitive on hydrostatic pressure and show quite unexpected effects. The at ambient pressure superconducting FeSe exhibits at pressures above $1 \mathrm{GPa}$ a phase, where superconductivity and antiferromagnetism seem to coexist on an atomic scale. The antiferromagnetic FeTe, on the other hand, transforms from a low pressure antiferromagnet to a ferromagnet above $2 \mathrm{GPa}$. These pressure studies underline the close relation of the electronic phases to the crystallographic properties, since in these simple compounds already minor changes in the structure for example by application of pressure lead to a drastic change of the superconducting and magnetic properties.
\end{abstract}

M. Bendele ( $\varangle)$

Dipartimento di Fisica, Universitá di Roma "La Sapienza",

P. le Aldo Moro 2, 00185 Roma, Italy

e-mail: markus.bendele@physik.uzh.ch

M. Bendele

Rome International Center for Materials Science Superstripes (RICMASS), Via dei Sabelli 119A, 00185 Roma, Italy

E. Pomjakushina $\cdot$ K. Conder

Laboratory for Developments and Methods, Paul Scherrer

Institute, 5232 Villigen PSI, Switzerland

R. Khasanov

Laboratory for Muon Spin Spectroscopy, Paul Scherrer Institute, 5232 Villigen PSI, Switzerland

H. Keller

Physik-Institut der Universität Zürich, Winterthurerstrasse 190, 8057 Zürich, Switzerland
Keywords Fe-based high temperature superconductors . Muon spin rotation - Pressure effect

In the year 2008, Kamihara et al. discovered superconductivity in the iron based compound $\mathrm{LaFeAsO}_{1-x} \mathrm{~F}_{x}$ with a superconducting transition temperature $T_{\mathrm{c}} \simeq 26 \mathrm{~K}[1]$. Remarkably, by replacing the nonmagnetic La by magnetic rare earth metals such as $\mathrm{Sm}, \mathrm{Nd}$, or $\mathrm{Ce}$, the transition temperature $T_{\mathrm{c}}$ even increased further to its current highest value of $\simeq 56 \mathrm{~K}$ in the Fe-based compounds [2]. The materials with the highest $T_{\mathrm{c}}$ 's differ from the ones with a lower $T_{\mathrm{c}}$ in a sense that application of external hydrostatic pressure leads to a decrease of $T_{\mathrm{c}}$ whereas the compounds with the lower $T_{\mathrm{c}}$ show rather an increase. This leads to the tentative observation of a possible optimal crystal configuration [3].

Shortly after the discovery of the high $T_{\mathrm{c}}$ 's in the 1111 family of Fe-based superconductors, other families with similar electronically active FeAs layers were found. The $\mathrm{Ba}_{1-x} \mathrm{~K}_{x} \mathrm{Fe}_{2} \mathrm{As}_{2}$ compound in the body-centered-tetragonal $\mathrm{ThCr}_{2} \mathrm{Si}_{2}$ (122-type) structure shows so far a maximum of $T_{\mathrm{c}} \simeq 38 \mathrm{~K}$ [4]. These simpler systems consist of the same electronically active FeAs layers that are separated by a single layer consisting of one atom only, whereas the 1111 compounds have separation layers of typically two types of atoms.

Even the binary compound $\mathrm{FeSe}_{1-x}$ (11-type) with a similar layered structure, yet without separating atoms, was found to be superconducting at $T_{\mathrm{c}} \simeq 8 \mathrm{~K}[5,6]$. Thus, from the first point of view, it could be argued that $\mathrm{FeSe}_{1-x}$ is not a high temperature superconductor since $T_{\mathrm{c}}$ is that low. Shortly after, however, it was found that $T_{\mathrm{c}}$ is very sensitive on chemical (substitution of Se by isovalent Te or $\mathrm{S}$ ) $[7,8]$ or hydrostatic pressure and reaches values of $36 \mathrm{~K}$ 
at $p \simeq 9 \mathrm{GPa}$ demonstrating that $\mathrm{FeSe}_{1-x}$ is in fact a high temperature superconductor $[9,10]$.

A more detailed investigation of $T_{\mathrm{c}}(p)$ with finer pressure steps at low pressures up to $\sim 2.5 \mathrm{GPa}$ revealed a nonlinear increase $[11,12]$. It exhibits a local maximum at $p \simeq 0.8 \mathrm{GPa}$ followed by a decrease of $T_{\mathrm{c}}$ to a local minimum at $p \simeq 1.2 \mathrm{GPa}$. At higher pressures $(p \geq 1.2 \mathrm{GPa})$, $T_{\mathrm{c}}$ increases again. Yet, for all pressures, the magnetization measurements revealed that the samples are bulk superconductors with a superconducting volume fraction of $\simeq 100 \%$.

The magnetic response of $\mathrm{FeSe}_{1-x}$ under pressure was studied in zero field $(\mathrm{ZF})$ muon spin rotation $(\mu \mathrm{SR})$ experiments. In such experiments, spin polarized muons are implanted into the sample. By monitoring the time evolution of the muon spin polarization, information on the local magnetic field at the muon stopping site $B_{\text {int }}$ and the magnetic volume fraction are obtained. Hereby, three different pressure regions with different magnetic states emerge in $\mathrm{FeSe}_{1-x}$. In the low-pressure region $(0 \leq p \lesssim 0.8 \mathrm{GPa})$ where $T_{\mathrm{c}}$ increases almost linear with $p$ (see Fig. 1), no static magnetic ordering was observed to the lowest temperatures investigated $(T \simeq 0.3 \mathrm{~K}$ ). For pressures above $\simeq 0.8 \mathrm{GPa}$, a spontaneous muon spin precession is observed indicating that static long range magnetic order on the $\mu \mathrm{SR}$ time scale is present below the antiferromagnetic Néel temperature $T_{\mathrm{N}}>T_{\mathrm{c}}$. The occurring magnetism is found to be of incommensurate nature. As soon as superconductivity emerges, however, the magnetic order is suppressed with decreasing temperature. This is reflected in the temperature dependence of the internal magnetic field $B_{\text {int }}$ and in the magnetic volume fraction that are both suppressed (see Fig. 1). However, with increasing pressure, this effect becomes less pronounced, and finally at pressures above $p \geq 1.2 \mathrm{GPa}$ the magnetic volume fraction reaches $100 \%$ and stays in bulk even in the superconducting state. Also, the magnetic order parameter $B_{\text {int }}$ is not decreasing in the superconducting state any more. At the same time, the magnetic order becomes commensurate. With increasing pressure both $B_{\text {int }}$ and $T_{\mathrm{N}}$ continue to increase up to the highest pressure investigated (Fig. 1) $[11,12]$. In addition to $\mu \mathrm{SR}$, indications of magnetic ordering at high pressures has been observed in NMR experiments with comparable ordering temperatures [13].

Keeping in mind that the superconducting volume fraction is $100 \%$ for all pressures investigated and that the magnetic volume fraction reaches $100 \%$ at pressures above 1.2 GPa indicates that both ground states coexist in the whole sample volume. The data does not show any signature for macroscopic phase separation into superconducting and magnetic regions bigger than a few $\mathrm{nm}$ size, as observed, e.g., in $A_{1-x} \mathrm{Fe}_{2-y} \mathrm{Se}_{2}$ [14]. Moreover, in the binary FeSe no sublattice similar to $\mathrm{Ce}$ - or $\mathrm{Sm} 1111$ is present that could order magnetically. In these compounds the rare earth atoms, $\mathrm{Ce}$ or Sm, exhibit static antiferromagnetic ordering while superconductivity takes place in the FeAs layers $[15,16]$. All

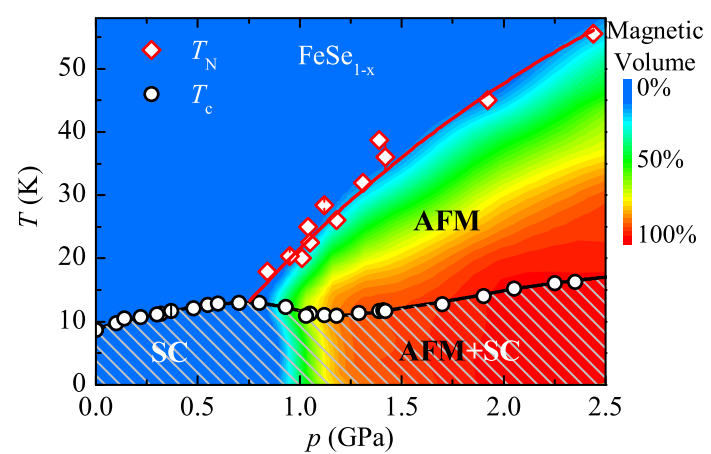

Fig. 1 Pressure dependence of the superconducting transition temperature $T_{\mathrm{c}}$, the magnetic ordering temperature $T_{\mathrm{N}}$, and the superconducting and magnetic volume fractions (the superconducting volume is $100 \%$ for all pressures investigated) obtained by ac susceptibility and muon spin rotation experiments for $\mathrm{FeSe}_{1-x}$. The lines marking the pressure dependence of $T_{\mathrm{c}}$ and $T_{\mathrm{N}}$ are guides to the eyes. $\mathrm{SC}$ and AFM denote the superconducting and magnetic states of the samples, respectively. The nonmagnetic state is indicated by the blue area (after [12])

these findings point rather to an atomic coexistence of the order parameters. Furthermore, it seems that the two ground states stabilize each other with pressure, since $T_{\mathrm{c}}, T_{\mathrm{N}}$, and $B_{\text {int }}$ are all increasing hand-in-hand with increasing pressure.

As already mentioned above, application of hydrostatic pressure is not the only possibility to increase $T_{\mathrm{c}}$ in the 11system [7, 8]. Substitution of Se by the isovalent elements Te or $\mathrm{S}$ leads to an increase of $T_{\mathrm{c}}$ up to $\simeq 14 \mathrm{~K}$ at a substitution level of $\approx 50 \%$ Se by Te $[7,8,17]$. A further increase of the Te content leads to the appearance of magnetic order in the system, whereas superconductivity and magnetism appear to coexist also here on atomic length scale. At high dopings, however, mainly incommensurate magnetic order is present with traces of superconductivity [8]. At which substitution level of Te the transition from superconductivity to magnetism occurs depends strongly on several further parameters, e.g., the Fe content in the system [18]. Nevertheless, with increasing amount of Te in the system $T_{\mathrm{N}}$ is increasing. For a Te concentration $\gtrsim 0.9$, no traces of superconductivity are detected in the system and the magnetic order changes to commensurate long range antiferromagnetism. In pure $\mathrm{FeTe}$ $T_{\mathrm{N}} \simeq 70 \mathrm{~K}$ and coincides with a structural transition making the phase transition first-order-like with a hysteretic behavior [8].

Application of hydrostatic pressure leads also in the antiferromagnetic FeTe to a rather unexpected behavior [19]: around $2 \mathrm{GPa}$ the system changes from a commensurate antiferromagnetic low pressure phase to a high pressure ferromagnetic one. This has been observed by means of neutron depolarization experiments, $\mu \mathrm{SR}$, and magnetization measurements.

Neutron transmission measurements reveal that at low pressures the polarization remains constant until the lowest 


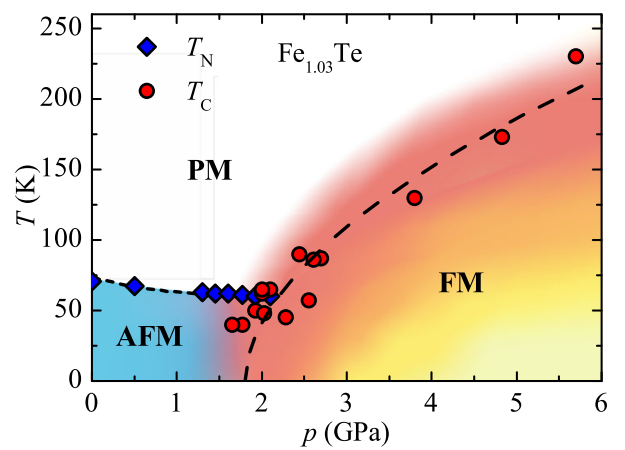

Fig. 2 Pressure dependence of the magnetic ordering temperatures $T_{\mathrm{N}}$ and $T_{\mathrm{C}}$ of FeTe. AFM denotes the antiferromagnetic, FM the ferromagnetic, and PM the paramagnetic state. Above $p \sim 2 \mathrm{GPa}, \mathrm{FeTe}$ becomes a bulk ferromagnet. In the crossover region from the AFM to the FM phase, the antiferromagnetic order is distorted due to a possible reorientation of the spin clusters, also seen in the PM state before the ferromagnetic order is fully established. The dashed lines indicating $T_{\mathrm{N}}(p)$ and $T_{\mathrm{C}}(p)$ are guides to the eyes (after [19]) (Color figure online)

temperature investigated. This clearly shows that the sample at ambient pressure is not ferromagnetic, but antiferromagnetic only; otherwise, the polarized neutrons would lose their polarization after passing through randomly aligned ferromagnetic domains, since the polarization of the beam would rotate with the Larmor precession. At high pressures, however, the neutron beam loses its polarization, which is directly showing the appearance of ferromagnetic domains in FeTe [19].

Additional field-dependent magnetization measurements revealed for a ferromagnet typical hysteretic behavior above $p \simeq 1.7 \mathrm{GPa}$, whereas with increasing pressure, this behavior is getting more pronounced. From these measurements, the magnetic moment can be extracted revealing a continuous increase with increasing pressure. At the maximum pressure investigated, the magnetic moment reaches large values of $3 \mu_{B}$ per $\mathrm{Fe}$ atom giving already a clear indication that the occurring ferromagnetism has bulk character, and thus is occupying the whole sample volume. The evolution of the ferromagnetism with pressure was further investigated from temperature dependent magnetization measurements revealing a continuous increase of the Curie temperature $T_{\mathrm{C}}$ (see Fig. 2) [19].

A microscopic view was obtained by means of ZF $\mu$ SR measurements. At low pressures, the spectra reveal the presence of commensurate antiferromagnetic order. Around the transition to the ferromagnetic state, however, a drastic change in the microscopic magnetic structure is observed. First, around 1.5 GPa, the commensurate AFM state changes to an incommensurate, then at slightly higher pressures around $1.8 \mathrm{GPa}$ an additional frequency occurs in the $\mu \mathrm{SR}$ signal at low temperatures that is ascribed to ferromagnetism. Increasing the pressure above $2 \mathrm{GPa}$ leads to a vanishing of the AFM signal and only the FM phase seems to be present. This change from the commensurate AFM to the FM phase seems gradual since FeTe shows a pressure region where both antiferromagnetism and ferromagnetism are coexisting in the sample.

Interestingly, the onset of the change in the magnetic order coincides with an observed structural transition from a tetragonal to a collapsed tetragonal phase [20], leading to the speculation that the occurring ferromagnetism might be strongly related to slight crystallographic changes with a possibly significantly different Fermi surface. On the other hand, in the superconducting FeSe, no crystallographic distortion was observed until now around the pressures where the AFM ordering develops.

These studies underline the close relation of the superconducting and magnetic properties with the crystal structure, since already minor changes in the parameters like application of small pressures lead to drastic changes in the electronic properties.

Acknowledgement M.B. acknowledges the financial support of the Swiss National Science Foundation (grant number PBZHP2_143495).

\section{References}

1. Kamihara, Y., Watanabe, T., Hirano, M., Hosono, H.: J. Am. Chem. Soc. 130, 3296 (2008)

2. Johnston, D.C.: Adv. Phys. 59, 803 (2010)

3. Mizuguchi, Y., Hara, Y., Deguchi, K., Tsuda, S., Yamaguchi, T., Takeda, K., Kotegawa, H., Tou, H., Takano, Y.: Supercond. Sci. Technol. 23, 054013 (2010)

4. Rotter, M., Tegel, M., Johrendt, D.: Phys. Rev. Lett. 101, 107006 (2008)

5. Hsu, F.C., Luo, J.Y., Yeh, K.-W., Chen, T.-K., Huang, T.-W., Wu, P.-M., Lee, Y.-C., Huang, Y.-L., Chu, Y.-Y., Yan, D.-C., Wu, M.-K.: Proc. Natl. Acad. Sci. USA 105, 14262 (2008)

6. Pomjakushina, E., Conder, K., Pomjakushin, V., Bendele, M., Khasanov, R.: Phys. Rev. B 80, 024517 (2009)

7. Sales, B.C., Sefat, A.S., McGuire, M.A., Jin, R.Y., Mandrus, D., Mozharivskyj, Y.: Phys. Rev. B 79, 094521 (2009)

8. Khasanov, R., Bendele, M., Amato, A., Babkevich, P., Boothroyd, A.T., Cervellino, A., Conder, K., Gvasaliya, S.N., Keller, H., Klauss, H.-H., Luetkens, H., Pomjakushin, V., Pomjakushina, E., Roessli, B.: Phys. Rev. B 80, 140511(R) (2009)

9. Margadonna, S., Takabayashi, Y., McDonald, M.T., Kasperkiewicz, K., Mizuguchi, Y., Takano, Y., Fitch, A.N., Suard, E., Prassides, K.: Chem. Commun. 5607 (2008)

10. Medvedev, S., McQueen, T.M., Troyan, I.A., Palasyuk, T., Eremets, M.I., Cava, R.J., Naghavi, S., Casper, F., Ksenofontov, V., Wortmann, G., Felser, C.: Nat. Mater. 8, 630 (2009)

11. Bendele, M., Amato, A., Conder, K., Elender, M., Keller, H., Klauss, H.-H., Luetkens, H., Pomjakushina, E., Raselli, A., Khasanov, R.: Phys. Rev. Lett. 104, 087003 (2010)

12. Bendele, M., Ichsanow, A., Pashkevich, Y., Keller, L., Strässle, T., Gusev, A., Pomjakushina, E., Conder, K., Khasanov, R., Keller, H.: Phys. Rev. B 85, 064517 (2012)

13. Imai, T., Ahilan, K., Ning, F.L., McQueen, T.M., Cava, R.J.: Phys. Rev. Lett. 102, 177005 (2009)

14. Ricci, A., Poccia, N., Campi, G., Joseph, B., Arrighetti, G., Barba, L., Reynolds, M., Burghammer, M., Takeya, H., Mizuguchi, Y., Takano, Y., Colapietro, M., Saini, N.L., Bianconi, A.: Phys. Rev. B 84, 060511(R) (2011) 
15. Maeter, H., Luetkens, H., Pashkevich, Y.G., Kwadrin, A., Khasanov, R., Amato, A., Gusev, A.A., Lamonova, K.V., Chervinskii, D.A., Klingeler, R., Hess, C., Behr, G., Büchner, B., Klauss, H.-H.: Phys. Rev. B 80, 094524 (2009)

16. Khasanov, R., Luetkens, H., Amato, A., Klauss, H.-H., Ren, Z.-A., Yang, J., Lu, W., Zhao, Z.-X.: Phys. Rev. B 78, 092506 (2008)

17. Bendele, M., Weyeneth, S., Puzniak, R., Maisuradze, A., Pomjakushina, E., Conder, K., Pomjakushin, V., Luetkens, H., Katrych, S., Wisniewski, A., Khasanov, R., Keller, H.: Phys. Rev. B 81, 224520 (2010)
18. Bendele, M., Babkevich, P., Katrych, S., Gvasaliya, S.N., Pomjakushina, E., Conder, K., Roessli, B., Boothroyd, A.T., Khasanov, R., Keller, H.: Phys. Rev. B 82, 212504 (2010)

19. Bendele, M., Maisuradze, A., Roessli, B., Gvasaliya, S.N., Pomjakushina, E., Weyeneth, S., Conder, K., Keller, H., Khasanov, R.: Phys. Rev. B 87, 060409(R) (2013)

20. Koz, C., Rößler, S., Tsirlin, A.A., Kasinathan, D., Börrnert, C., Hanfland, M., Rosner, H., Wirth, S., Schwarz, U.: Phys. Rev. B 86, 094505 (2012) 